\title{
Propuesta tecnológica para incrementar la productividad en la construcción masiva de vivienda
}

\author{
C.E. Arcudia-Abad, R.G. Solís-Carcaño y A.R. Cuesta-Santos \\ Facultad de Ingeniería, Universidad Autónoma de Yucatán \\ E-mail:aabad@tunku.uady.mx
}

(Recibido: agosto de 2005; aceptado: noviembre de 2006)

\begin{abstract}
Resumen
Se desarrolló una tecnología para incrementar proactivamente la productividad del recurso humano, particularmente para la construcción de vivienda en Yucatán. El modelo teórico considera el efecto de cada uno de los tres tipos de factores: los de insumo, los de proceso y los del contexto que rodea al sitio donde se realiza la obra. El procedimiento de aplicación consta de dos etapas principales: la creación de una base de datos y el análisis de nuevos proyectos. En la primera, se selecciona una actividad de construcción y se recaba la información básica sobre los indicadores de los factores y de la productividad, incluyendo el número necesario de proyectos para poder aplicar el análisis estadístico. En la segunda, ya constituida la base se pueden tratar los nuevos casos en los que se podrán tomar decisiones proactivas para modificar la productividad. Para validar la tecnología se analizaron dos proyectos de construcción de vivienda. En ellos se pudieron contrastar las diferencias en los resultados entre tomar la decisión de actuar proactivamente y no tomarla. Los resultados de la productividad obtenidos de ambos proyectos fueron diferentes, siendo superiores en el caso en donde se tomó la decisión de adoptar una conducta proactiva.
\end{abstract}

Descriptores: Productividad, construcción, gestión de recursos humanos, tecnología, vivienda, toma de decisiones.

\begin{abstract}
A technology was de vel oped to proactively in crease the produc tiv ity of the hu man resource, partic ularlyforhousing construction in Yucatan. The theoret ical model con siders theeffect of each one of the three types of fac tors, those of: in put, pro cess, and the con text that surrounds to the site where the work is made. The ap plica tion pro ce dure con sists of two main stages: the cre ation of data base and anal y sis of new pro jects. In the first one, a con struct ion ac tiv ity is selected and the ba sic in for ma tion on the in dica tors offac tors and the pratuc tivity, in clud ing the num ber nec es sary of pro jects to be able to ap ply the sta tis tical analy sis, is gathered. In the sec ond one, af ter the base is ready, the new cases can be treated in which it is possible to make proactivedecisions to mod ify the productivity. In or der to val idate the technol ogy two pro jects of hous ing con struc tion were an a lyzed. In them, the differ ences in the results be tween making or not proactive de ci sions could be con trasted. The produc tiv ity results ob tained in both pro jects were differ ent, be ing su pe rior those of the case in where the de ci sion to adopt a proactive con duct was taken.
\end{abstract}

Keywords: Productivity, construction, hu man resources manage ment, technology, hous ing, decision making. 


\section{Introducción}

A nivel nacional existe un rezago en la satisfacción de la demanda de vivienda, que de acuerdo con el último Censo Nacional de Población y Vivienda realizado en el 2000, alcanzó la cifra de 4 millones 290 mil 665 viviendas (INEGI). Entre los problemas asocia- dos a esta rezago está la poca eficiencia en el empleo de los recursos en la construcción, en particular el del humano, pues de acuerdo con estudios realizados en el Estado de Yucatán, el porcentaje del contenido básico del trabajo es en promedio 24,84\% (Alcudia et al., 2000); (Corona et al., 2000). De ahí la importancia de desarrollar tecnologías para gestionar el recurso humano de la construcción masiva de vivienda, con el objeto de incrementar su productividad.

En el presente trabajo se describe una propuesta tecnológica para incrementar la productividad del recurso humano en la construcción de vivienda de interés social. Esta tecnología se fundamentó en el enfoque de sistemas y procesos. De acuerdo con él, la productividad resultante o productividad acumulada está influida por tres tipos de factores: los de insumo, los de proceso y los del contexto (Figura 1).

Dada la uniformidad de los proyectos de vivienda de interés social en el estado, solo se observó un factor de insumo que fue el grado de complejidad del diseño a ejecutar (GC), el cual se obtuvo del análisis de los planos y especificaciones. Hubo dos conjuntos de factores de proceso, uno de ellos fue la capacidad del recurso humano (CRH) y el otro la administración del proyecto (AP). El factor de contexto que se incluyó fue el de las condiciones meteorológicas representadas por el índice del clima (IC), por haber indicios en la literatura de ser un factor de cierta variabilidad (Thomas, 1999).

Los datos de productividad asociados con los factores son: el horizonte de productividad (HP), la productividad del proceso (PP), el umbral de la productividad (UP), el horizonte de productividad calculado (HPC), la productividad acumulada total (PAT) y el índice de anormalidad (IA). El HP es la mejor productividad que se obtiene en el proyecto; la PP es la pérdida que sufre la productividad por el efecto de los factores de la administración y del contexto; el UP es el límite que nos indica cuál debe ser el valor de la productividad diaria para ser considerada dentro de lo aceptable, en un proyecto es igual a la mitad del horizonte de productividad calculado para un grado de complejidad específico; el HPC es el valor de la mejor productividad de la base de datos que incluye los datos de los proyectos estudiados, del cual se pueden determinar los horizontes

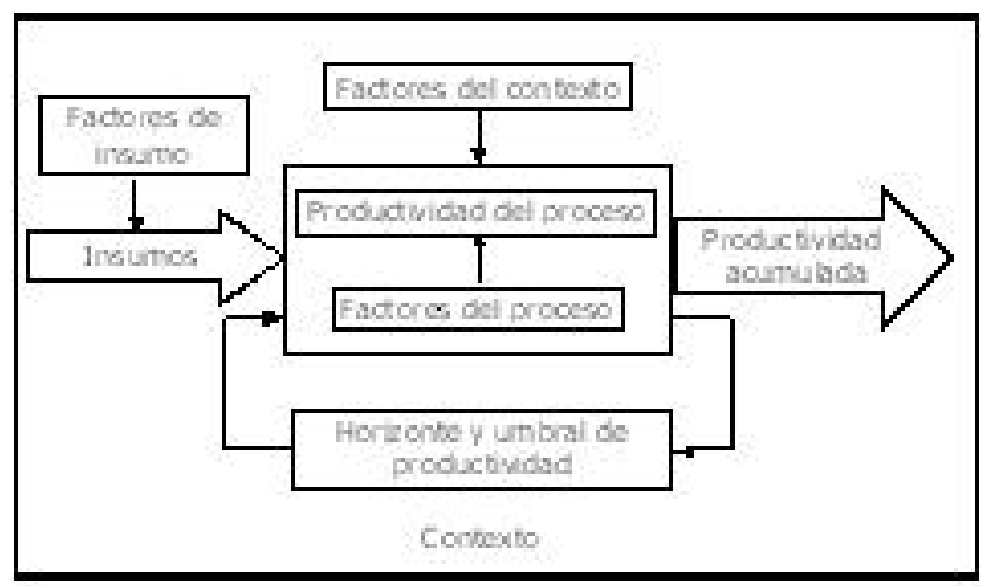

Figura 1. Modelo Conceptual 
de productividad por grado de complejidad, particularmente (HPCn); el PAT es el cociente de la producción diaria acumulada entre las horas hombre acumuladas; y el IA es el cociente de dividir el número de días anormales entre el total de días hábiles en que se realizó la actividad estudiada en un proyecto (entendiéndose por día anormal aquel en el que la productividad está por debajo del UP).

El objetivo de la investigación fue desarrollar una propuesta tecnológica que permita tomar decisiones para regular proactivamente la productividad con el fin de incrementarla, considerando los tres tipos de factores que en ella influye. Esta propuesta fue desarrollada con base en una revisión sobre las teorías actuales sobre la productividad en la construcción, y mediante un método que busca no solo conocer la realidad, sino transformarla mediante la actividad científica. Las técnicas utilizadas fueron: el desarrollo de un modelo conceptual, la integración de una base de datos soporte del modelo, el desarrollo de un proce- dimiento de aplicación y la validación de la propuesta. El resultado de esta investigación tal como se obtuvo y se presenta en este artículo puede ser aplicable a la construcción en serie de viviendas financiadas con créditos de interés social y que utilicen los mismos procesos constructivos.

\section{Procedimiento de aplicación de la tecnología}

Para una actividad dada de construcción el procedimiento de aplicación de la tecnología es el siguiente:

- Construcción de la base de datos

- Captura de datos de los proyectos

* Identificación de los factores de insumo
* Cálculo del horizonte de produc tividad

* Cálculo de los factores del contexto

- $\quad$ Integración de la base de datos

* Cálculo del horizonte de productividad calculado y del umbral de productividad

* Cálculo del índice de anormalidad

* Cálculo de los factores del contexto

* Cálculo de los factores del proceso

* Cálculo de la productividad acumulada

* Cálculo de la productividad del proceso

- $\quad$ Análisis de un nuevo proyecto

- Determinación y cálculo de los indicadores de los factores y de productividad

- Comparación proactiva de los indicadores con los de la base de datos y análisis de incidencias

- $\quad$ Evaluación final de los resultados del proyecto

- Incorporación de los datos a la base

\section{Validación de la tecnología}

La actividad de construcción seleccionada para ilustrar la tecnología fue la elaboración de paredes de bloques de concreto. Ésta es 
una de las tres actividades con mayor costo (13\% del total) y ocupa el 19\% del tiempo de construcción de una unidad (Pech, 2002).

Aunque esta actividad es superada en costo por las de cimentación y acabados, técnicamente es la que presentó las características más aproximadas para enfocarse como un procedimiento industrial.

Se capturaron los datos de los 15 proyectos que constituyeron la base de datos original. Los grados de complejidad incluyeron principalmente la uniformidad de los ejes de trazo y la densidad de los elementos rigidizantes de concreto, mismos que están incluidos en la tabla 1. La productividad diaria se calculó utilizando la ecuación 1, en donde la cantidad de actividad realizada se mide en la unidad física corres- pondiente $\left(\mathrm{m}, \mathrm{m}^{2}, \mathrm{~m}^{3}\right.$, etc.), y el recurso humano utilizado para la realizar la actividad se expresa en horas - hombre $(h-h)$.

$$
P D=\frac{\text { Cantid ad deactividad realizada }}{h-h}
$$

Como ejemplo, en la tabla 2 se presentan los datos de productividad diaria del proyecto 101. Con estos datos se obtuvo el horizonte de productividad de cada proyecto (HP), determiado por la mediana del los cinco días en los cuales se observó la mejor productividad. El resultado del horizonte de productividad para el proyecto 101 fue de 1.4375 $\mathrm{m}^{2} / \mathrm{h}-\mathrm{h}$.

De este mismo modo, se calcularon los horizontes de productividad de los demás proyectos obteniéndose los resultados incluidos en la tabla 3. Se calcularon y determinaron todos los factores y los datos de productividad. Se integró así la primera base de datos necesaria para la aplicación proactiva de la tecnología. Las tablas 4 y 5 incluyen los indicadores básicos de la base de datos. En ellas se han indexado las variables: capacidad del recurso humano, administración del proyecto y productividad del proceso, dividiendo sus valores entre el horizonte de productividad calculado para el grado de complejidad 1; (HPC1); los índices

Tabla 1. Grado de complejidad y código de proyecto

\begin{tabular}{ccc}
\hline Proyecto & GC & Código \\
\hline 1 & 1 & 101 \\
2 & 1 & 102 \\
3 & 1 & 103 \\
4 & 1 & 104 \\
5 & 1 & 105 \\
6 & 1 & 106 \\
7 & 1 & 107 \\
8 & 1 & 108 \\
9 & 1 & 109 \\
10 & 1 & 110 \\
11 & 1 & 111 \\
12 & 1 & 112 \\
13 & 2 & 201 \\
14 & 2 & 202 \\
15 & 3 & 301 \\
\hline
\end{tabular}


DOI: http://dx.doi.org/10.22201/fi.25940732e.2007.08n2.006

C.E. Arcudia-Abad, R.G. Solís-Carcaño y A.R. Cuesta-Santos

Tabla 2. Datos de productividad diaria del proyecto 101

\begin{tabular}{cccccccc}
\hline Días & $\begin{array}{c}\text { Productividad } \\
\text { diaria } \mathrm{m} h \mathrm{~h} h\end{array}$ & Días & $\begin{array}{c}\text { Productividad } \\
\text { diaria } \mathrm{m} h-\mathrm{h}\end{array}$ & Días & $\begin{array}{c}\text { Productividad } \\
\text { diaria } \mathrm{m}^{2} h-\mathrm{h}\end{array}$ & Días & $\begin{array}{c}\text { Productividad } \\
\text { diaria } \mathrm{m} / \mathrm{h}-\mathrm{h}\end{array}$ \\
\hline 1 & 0,5740 & 7 & 1,2750 & 13 & 1,4375 & 19 & 1,2938 \\
2 & 1,0540 & 8 & 1,4840 & 14 & 0,0000 & 20 & 0,0000 \\
3 & 0,0000 & 9 & 0,3189 & 15 & 0,0000 & 21 & 0,7667 \\
4 & 0,9563 & 10 & 1,5000 & 16 & 0,0000 & 22 & 1,0542 \\
5 & 0,9444 & 11 & 0,9138 & 17 & 0,0000 & 23 & 0,4348 \\
6 & 1,0625 & 12 & 0,6893 & 18 & 1,0063 & & \\
\hline
\end{tabular}

Tabla 3. Horizontes de productividad

\begin{tabular}{cc}
\hline Proyecto & Horizonte de productividad \\
\hline 101 & 1,44 \\
102 & 2,25 \\
103 & 2,46 \\
104 & 1,33 \\
105 & 1,91 \\
106 & 1,88 \\
107 & 2,53 \\
108 & 1,85 \\
109 & 1,60 \\
110 & 2,40 \\
111 & 2,18 \\
112 & 1,75 \\
201 & 1,80 \\
202 & 1,05 \\
301 & 0,88 \\
\hline
\end{tabular}

Tabla 4. Valores de los factores obtenidos en los 15 proyectos (continúa ...)

\begin{tabular}{cccccccc}
\hline \multicolumn{7}{c}{ Insumo } & \multicolumn{9}{c}{ Tipos de factores } \\
\hline Ident. & GC & CRH & ICRH & AP & IAP & IA & IC \\
\hline 101 & 1 & 0,55 & 0,28 & $-0,2$ & $-0,01$ & 0,61 & 0,00 \\
102 & 1 & $-0,26$ & $-0,13$ & 0,88 & 0,44 & 0,57 & 0,00 \\
103 & 1 & $-0,47$ & $-0,24$ & 1,3 & 0,65 & 0,59 & 0,00 \\
104 & 1 & 0,66 & 0,33 & $-0,52$ & $-0,26$ & 0,47 & 0,00 \\
105 & 1 & 0,08 & 0,04 & 0,26 & 0,13 & 0,25 & 0,25 \\
106 & 1 & 0,11 & 0,06 & 0,57 & 0,29 & 0,47 & 0,00 \\
107 & 1 & $-0,54$ & $-0,27$ & 1,39 & 0,70 & 0,14 & 0,18 \\
\hline
\end{tabular}


DOI: http://dx.doi.org/10.22201/fi.25940732e.2007.08n2.006

Propuesta tecnológica para incrementar la productividad en la construcción masiva de vivienda

Tabla 4. Valores de los factores obtenidos en los 15 proyectos (... continúa ción)

\begin{tabular}{cccccccc}
\hline \multicolumn{7}{c}{} & \multicolumn{7}{c}{ Tipos de factores } \\
\hline \multicolumn{2}{c}{ Insumo } & CR & \multicolumn{1}{c}{ Proceso } & \multicolumn{2}{c}{ Contexto } \\
\hline Ident. & GC & CRH & ICRH & AP & IAP & IA & IC \\
\hline 108 & 1 & 0,14 & 0,07 & 0,58 & 0,29 & 0,59 & 0,19 \\
109 & 1 & 0,39 & 0,20 & $-0,04$ & $-0,02$ & 0,56 & 0,00 \\
110 & 1 & $-0,41$ & $-0,21$ & 1,49 & 0,75 & 0,43 & 0,02 \\
111 & 1 & $-0,19$ & $-0,10$ & 1,18 & 0,59 & 0,46 & 0,02 \\
112 & 1 & 0,24 & 0,12 & 0,41 & 0,21 & 0,47 & 0,03 \\
201 & 2 & $-0,36$ & $-0,18$ & 0,93 & 0,47 & 0,07 & 0,00 \\
202 & 2 & 0,39 & 0,20 & $-0,21$ & $-0,11$ & 0,14 & 0,00 \\
301 & 3 & 0,01 & 0,01 & 0,09 & 0,05 & 2,42 & 0,00 \\
\hline
\end{tabular}

Tabla 5. Valores de productividad obtenidos en los 15 proyectos

\begin{tabular}{cccccccc}
\hline PROYECTO & HP & HPC & PAT & UP & PP & IPP & PPC \\
\hline 101 & 1,44 & 1,99 & 0,90 & 0,995 & 0,54 & 0,27 & 0,54 \\
102 & 2,25 & 1,99 & 1,63 & 0,995 & 0,62 & 0,31 & 0,62 \\
103 & 2,46 & 1,99 & 1,63 & 0,995 & 0,83 & 0,42 & 0,83 \\
104 & 1,33 & 1,99 & 1,19 & 0,995 & 0,14 & 0,07 & 0,13 \\
105 & 1,91 & 1,99 & 1,57 & 0,995 & 0,34 & 0,14 & 0,33 \\
106 & 1,88 & 1,99 & 1,20 & 0,995 & 0,68 & 0,34 & 0,68 \\
107 & 2,53 & 1,99 & 1,68 & 0,995 & 0,85 & 0,43 & 0,85 \\
108 & 1,85 & 1,99 & 1,13 & 0,995 & 0,72 & 0,36 & 0,72 \\
109 & 1,60 & 1,99 & 1,25 & 0,995 & 0,35 & 0,18 & 0,34 \\
110 & 2,40 & 1,99 & 1,32 & 0,995 & 1,08 & 0,54 & 1,08 \\
111 & 2,18 & 1,99 & 1,19 & 0,995 & 0,99 & 0,50 & 0,99 \\
112 & 1,75 & 1,99 & 1,10 & 0,995 & 0,65 & 0,33 & 0,65 \\
201 & 1,80 & 1,44 & 1,23 & 0,720 & 0,57 & 0,29 & 0,56 \\
202 & 1,05 & 1,44 & 0,87 & 0,720 & 0,18 & 0,09 & 0,17 \\
301 & 0,88 & 0,89 & 0,78 & 0,445 & 0,10 & 0,05 & 0,09 \\
\hline
\end{tabular}

resultantes fueron: índice de capacidad del recurso humano (ICRH), índice de administración del proyecto (IPP) y productividad del proceso (IAP).

Para la aplicación de la fase proactiva de la tecnología se analizaron simultáneamente dos proyectos más, el 113 y el 203. La tabla 6 incluye los resultados obtenidos en ambos proyectos a los seis días de haberse iniciado cada uno de ellos.

En el proyecto 113, aunque hubo conocimiento de resultados insatisfactorios por parte de la empresa, así como de los subcontratistas y de los trabajadores, no se 
estableció compromiso alguno en cuanto actuar proactivamente en aras de elevar los niveles de productividad.

En el caso del proyecto 203, aunque en ese momento se pudo haber aceptado que la empresa se estaba desempeñando de manera aceptable, sus administradores pensaron que la situación podría mejorarse si se decidía actuar proactivamente en esa dirección. Los problemas que se observaron estuvieron asociados a la falta de organización del trabajo, el absentismo y la impuntualidad. De estos, el que provocó mayores incidencias en la productividad fue el absentismo de día lunes, motivado a que la mayoría de los trabajadores se desplazan desde otras comunidades para asistir a su centro de trabajo, y a que acostumbran ingerir bebidas alcohólicas en su día de descanso.
Para lo anterior, la empresa expuso los resultados obtenidos en los días iniciales del proyecto a los subcontratistas y obreros. Éstos estuvieron interesados en superar los resultados iniciales, proponiéndose como meta lograr una productividad acumulada de por lo menos un $10 \%$ por encima del valor obtenido en los seis primeros días.

Para lograr resolver los problemas, se identificaron las siguientes alternativas:

1. Declarar el lunes oficialmente como día de descanso y esforzarse más los cinco días restantes.

2. Trabajar los lunes y asumir el compromiso de asistir puntual y en buenas condiciones físicas al trabajo.

Tabla 6. Resultados en los primeros días

\begin{tabular}{|c|c|c|c|c|}
\hline \multirow{2}{*}{$\begin{array}{c}\text { Tipo de dato } \\
\text { Proyecto } \\
\end{array}$} & \multicolumn{4}{|c|}{ Valores a los 6 días } \\
\hline & \multicolumn{2}{|c|}{113} & \multicolumn{2}{|c|}{203} \\
\hline \multicolumn{5}{|l|}{ Factores } \\
\hline \multicolumn{5}{|l|}{ De insumo } \\
\hline GC & 1 & & 2 & \\
\hline \multicolumn{5}{|l|}{ De proceso } \\
\hline $\begin{array}{ll}-\quad \mathrm{CRH} \\
\end{array}$ & 0,82 & $\mathrm{~m}^{2} / \mathrm{h}-\mathrm{h}$ & $-0,60$ & $\mathrm{~m}^{2} / \mathrm{h}-\mathrm{h}$ \\
\hline ICRH & 0,41 & & 0,30 & \\
\hline $\mathrm{AP}$ & $-0,65$ & $\mathrm{~m}^{2} / \mathrm{h}-\mathrm{h}$ & 0,81 & $\mathrm{~m}^{2} \mathrm{~h}-\mathrm{h}$ \\
\hline IAP & $-0,33$ & & 0,41 & \\
\hline IA & 0,67 & & 0,17 & \\
\hline \multicolumn{5}{|l|}{ De contexto } \\
\hline$-\quad$ IC & 0,00 & $\mathrm{~m}^{2} / \mathrm{h}-\mathrm{h}$ & 0,00 & $\mathrm{~m}^{2} / \mathrm{h}-\mathrm{h}$ \\
\hline \multicolumn{5}{|l|}{ Productividad } \\
\hline $\begin{array}{ll}- & \mathrm{HP}\end{array}$ & 1,17 & $m^{2} / h-h$ & 2,04 & $\mathrm{~m}^{2} \mathrm{~h}-\mathrm{h}$ \\
\hline HPC & 1,99 & $\mathrm{~m}^{2} / \mathrm{h}-\mathrm{h}$ & 1,44 & $\mathrm{~m}^{2} / \mathrm{h}-\mathrm{h}$ \\
\hline PAT & 0,999 & $\mathrm{~m}^{2} / \mathrm{h}-\mathrm{h}$ & 1,83 & $\mathrm{~m}^{2} \mathrm{~h}-\mathrm{h}$ \\
\hline UP & 0,995 & $m^{2} / h-h$ & 0,72 & $m^{2} h-h$ \\
\hline $\mathrm{PP}$ & 0,17 & $\mathrm{~m}^{2} / \mathrm{h}-\mathrm{h}$ & 0,21 & $\mathrm{~m}^{2} \mathrm{~h}-\mathrm{h}$ \\
\hline
\end{tabular}


Para la alternativa 1 , si bien podrían los trabajadores lograr el desempeño trabajando arduo los cinco días hábiles de la semana, la compañía tendría que afrontar los costos indirectos de los días inhábiles. Por otra parte, para cumplir con la alternativa 2 los obreros pensaron que era necesario contar con el compromiso de todos ellos y de la administración la que debería establecer algunas regulaciones y apoyo en la organización del trabajo.

Se adoptó la alternativa 2, comprometiéndose los trabajadores a asistir los lunes y el residente a vigilar el orden en el sitio de trabajo, así como ayudar a los subcontratistas en la organización de las actividades sobre todo de los peones.

\section{Evaluación final de los resultados de los nuevos proyectos}

Los datos finales de productividad a los 18 días de los proyectos 113 y 203 se encuentran en la tabla 7. Para contrastarlos con los de la base de datos, se les realizaron las pruebas de hipótesis correspondientes. De ellas se obtuvo, para el proyecto 113, que estadísticamente la posición de los indicadores con referencia a los de la base de datos sigue siendo la misma. Para el proyecto 203 la posición de los indicadores con referencia a los de la base de datos sufrió algunos cambios. Los factores de capacidad del recurso humano y de anormalidad, que ya desde un principio estaban por debajo de la media de la base, permanecieron en esa posición. En

Tabla 7. Resumen de indicadores finales

\begin{tabular}{|c|c|c|c|c|}
\hline \multirow{2}{*}{$\begin{array}{c}\text { Tipo de dato } \\
\text { Proyecto }\end{array}$} & \multicolumn{4}{|c|}{ Valores a 18 días } \\
\hline & \multicolumn{2}{|c|}{113} & \multicolumn{2}{|c|}{203} \\
\hline \multicolumn{5}{|l|}{ Factores } \\
\hline \multicolumn{5}{|l|}{ De insumo } \\
\hline$-\quad \mathrm{GC}$ & 1 & & 2 & \\
\hline \multicolumn{5}{|l|}{ De proceso } \\
\hline $\mathrm{CRH}$ & 0,82 & $\mathrm{~m}^{2} / \mathrm{h}-\mathrm{h}$ & $-1,27$ & $\mathrm{~m}^{2} / \mathrm{h}-\mathrm{h}$ \\
\hline ICRH & 0,41 & & $-0,88$ & \\
\hline $\mathrm{AP}$ & $-0,65$ & $\mathrm{~m}^{2} / \mathrm{h}-\mathrm{h}$ & 0,92 & $m^{2} / h-h$ \\
\hline IAP & $-0,33$ & & 0,33 & \\
\hline IA & 0,67 & & 0,06 & \\
\hline \multicolumn{5}{|l|}{ De contexto } \\
\hline IC & 0,00 & $\mathrm{~m}^{2} / \mathrm{h}-\mathrm{h}$ & 0,00 & $\mathrm{~m}^{2} \mathrm{~h}-\mathrm{h}$ \\
\hline \multicolumn{5}{|l|}{ Productividad } \\
\hline$-\quad \mathrm{HP}$ & 1,32 & $\mathrm{~m}^{2} / \mathrm{h}-\mathrm{h}$ & 2,71 & $\mathrm{~m}^{2} / \mathrm{h}-\mathrm{h}$ \\
\hline HPC & 1,99 & $\mathrm{~m}^{2} / \mathrm{h}-\mathrm{h}$ & 1,44 & $m^{2} / h-h$ \\
\hline PAT & 1,01 & $\mathrm{~m}^{2} / \mathrm{h}-\mathrm{h}$ & 2,06 & $m^{2} / h-h$ \\
\hline UP & 0,99 & $\mathrm{~m}^{2} / \mathrm{h}-\mathrm{h}$ & 0,72 & $\mathrm{~m}^{2} / \mathrm{h}-\mathrm{h}$ \\
\hline $\mathrm{PP}$ & 0,30 & $\mathrm{~m}^{2} / \mathrm{h}-\mathrm{h}$ & 0,65 & $\mathrm{~m}^{2} / \mathrm{h}-\mathrm{h}$ \\
\hline
\end{tabular}


cuanto al índice de administración del proyecto que se encontraba en el nivel de la media de la base, subió por encima de ella, lo cual significó una mejora en el desempeño. Con referencia a los indicadores de productividad, todos se elevaron significativamente con referencia a la media de la base de datos. En el caso de la productividad acumulada total no se manifestó una variación estadística pues desde el inicio ya estaba por encima del promedio de la base.

\section{Discusión}

Ya otros autores han hecho propuestas para implementar cambios en la manera de gestionar el recurso humano de la construcción, así Maloney y Federle (1991), Sanders y Eskridge (1993), consideran tomar en cuenta para estos cambios la cultura de las organizaciones. Otros sugieren la necesidad de que en las compañías constructoras se desarrolle la creatividad entre los profesionales. Al respecto Farid et al. (1993) ilustran las ventajas competitivas que una constructora podría alcanzar desarrollando la creatividad en sus administradores.

Otro aspecto importante en la experiencia del proyecto 203 es el incremento del grado de involucramiento de los trabajadores. Esto coincide, de acuerdo con Khan (1993), con la necesidad de aplicar las teorías de la motivación a la gestión de los recursos humanos de la construcción, pues la participación en la toma de decisiones aumenta el nivel de motivación para aplicarlas.

La participación en la toma de decisiones mitiga el surgimiento de los conflictos en las situaciones del trabajo, lo cual tiene incidencia en el desempeño de las actividades, en este caso las de la construcción. Woodrich (1993) y Weston y Gibson (1993) confirman que esto se cumple por lo general cuando se adopta la asociación (partnering) como una técnica para evitar los conflictos de intereses.
En el caso específico del proyecto 203, la empresa decidió involucrar a los subcontratistas y trabajadores en la toma de decisiones relacionadas con el empleo del recurso humano; esto generalmente no es común en la industria de la construcción donde la organización es altamente jerárquica, por lo que significó el inicio de un cambio en la cultura de la empresa. En este contexto se aceptó la asociación fijando y compartiendo una meta en la búsqueda de la mejora en la productividad. Dadas las características del ambiente de trabajo, en esta ocasión no se adoptaron medidas directas para evaluar el grado de involucramiento y funcionamiento de la asociación; el incremento de la productividad obtenido, incluso más allá de la meta fijada, fue considerada como una medición indirecta de los dos aspectos mencionados.

\section{Conclusiones}

El incremento de la productividad alcanzado, lleva a concluir que la estrategia general de esta propuesta tecnológica puede aplicarse a otras actividades, a otros tipos de construcción e incluso a otros trabajos que se realicen con proyectos repetitivos y con brigadas $\mathrm{o}$ cuadrillas, para lo cual habrán de hacerse las adecuaciones pertinentes, debido a que la información básica específica necesaria puede cambiar.

En esta investigación la tecnología fue validada con dos casos, por lo que, aunque no se pueden hacer generalizaciones, en ellos se pudo observar que para actuar proactivamente la empresa debe adoptar una actitud de cambio de valores permitiendo la participación de los trabajadores en la toma de decisiones de sus propias actividades laborales.

El que se hayan dado las circunstancias propicias en la empresa del proyecto 203, no quiere decir que sus valores se hayan transformado totalmente y que de ahora en 
adelante vaya a actuar consistentemente de esta manera. El efecto observado puede deberse también en parte a la motivación desarrollada por los trabajadores y administradores respecto a involucrarse en hacer algo diferente.

No se debe perder de vista que el éxito de un proyecto de construcción no sólo depende del uso de tecnologías basadas en diseño, materiales y maquinarias, sino que también juega un papel importante las tecnologías administrativas, siendo primordial la de la gestión del recurso humano.

En México, generalmente se ha dado poca importancia a la investigación de los aspectos administrativos de la construcción, por lo que los autores esperan que los resultados aquí presentados contribuyan a que se de mayor reconocimiento y apoyo al desarrollo de esta línea de investigación, importante para el desarrollo y competitividad de este sector de la industria nacional.

\section{Agradecimientos}

Al FOSISIERRA del CONACYT por el apoyo financiero otorgado para realizar el proyecto "Determinación de los factores que afectan la productividad mano de obra de la construcción", clave 990401, con el cual se recolectó la información básica para el desarrollo de la tecnología.

\section{Referencias}

Arcudia A., Carlos E., José A. González F. y José H. Loría Arcila. (2000). Muestreo de trabajo en la construcción de vivienda de interés social. Ingeniería industrial (La Habana), XXI, No. 4, pp. 12-18.
Corona S., Gilberto, Carlos Arcudia A. y José Loría A. (2000). Estimación de la productividad en la construcción masiva de vivienda mediante el estudio del trabajo. Ingeniería: Revista académica de la Facultad de Ingeniería Universidad Autónoma de Yucatán (Mérida), 4, No. 3, pp. 25-40.

Farid F., Ahmed R. El-Sharkawy and Austin K.L. (1993). Managing for Creativity and Innovation in $\mathrm{A} / \mathrm{E} / \mathrm{C}$ organizations. Journal of Manage ment in Engineering (New York), 9, No. 4, pp. 399-409.

Khan M.S. (1993). Methods of Motivating for Increased Productivity. Journal of Management in Engineering (New York), 9, No. 2, pp. 148-155.

Maloney W.F. y Federle M.O. (1991). Organizational Culture and Manage ment. Journal of Management in Engineering (New York), 7, No. 1, pp. 43-57.

Pech-Pérez, J. G. (2002). Base de datos del SINCO. Universidad Autónoma de Yucatán, Mérida.

Sanders S.R. y Eskridge W.F. (1993). Managing Implementation of Change. Journal of Management in Engineering (New York), 9, No. 4, pp. 365-381.

Thomas R., D.R. Riley and Sanvido V.E. (1999). Loss of Labor Productivity due to Delivery Métodos and Weather. Journal of Construction Engineering and Management, 125, No. 1, pp. 39-46.

Weston D.C. and Gibson E. Jr. (1993). Part nering-Project Perfor mance in U.S. Army Corps of Engineers. Journal of Management in Engineering (New York), 9, No. 4, pp. 410-425.

Woodrich A. (1993). Partnering: Providing Effective Project Control. Journal of Management in Engineering, (New York), 9, No. 2, pp. 136-141. 
Semblanza de los autores

Carlos Enrique Arcudia-Abad. Es Ingeniero químico y maestro en educación superior por la Universidad Autónoma de Yucatán (UADY); y doctor en ciencias técnicas por el Instituto Superior Politécnico “José Antonio Echeverría". Ejerció su profesión por 14 años en el campo del control de calidad, producción e ingeniería de proyectos. Durante los últimos 14 años se ha dedicado a la investigación y docencia en el área de construcción, en la Facultad de Ingeniería de la UADY. Ha impartido las materias de metodología de la investigación, introducción a la ingeniería y programación y análisis de operaciones de construcción. Ha sido director de proyectos de investigación con financiamiento de la DIGSA, PIIES y CONACYT. Es autor de 25 publicaciones, en temas relacionados con productividad, gestión de recursos humanos en la construcción y educación para ingeniería.

Rómel G. Solís-Carcaño. Ingeniero civil y maestro en ingeniería por la Universidad Autónoma de Yucatán (UADY). Ejerció su profesión por 20 años en el campo de la supervisión de obra. En los últimos 6 años se ha dedicado a la investigación y docencia en la Facultad de Ingeniería de la UADY. Ha impartido las materias de cons trucción, edificación, planeación y control de proyectos; así como evaluación de proyectos. Ha sido director de dos proyectos de investigación con financiamiento del CONACYT. Es autor de 25 publicaciones en temas relacionados con tecnología del concreto, supervisión de obra e investigación educativa.

Armando Ramón Cuesta-Santos. Psicólogo, master en organización del trabajo y doctor en ciencias económicas por la Universidad de la Habana. Es profesor titular de la Facultad de Ingeniería Indus trial del Instituto Superior Politécnico "José Antonio Echeverría" y ostenta la Distinción por la Educación Cubana. Ha impartido materias relacionadas con la psicología organizacional, organización del trabajo y gestión de recursos humanos. Es coautor de 3 libros de texto, tres monografías y cuatro libros sobre diferentes aspectos de la gestión de recursos humanos. Ha publicado más de 50 artículos de carácter científico técnico en revistas, tanto de su país como extranjeras. 